\title{
The localization of HPV and CMV in the adipose tissues of female diabetic type I rats and the possibility of having a role of reactivity of COVID- 19 in diabetic subjects as a new medical hypothesis
}

\begin{abstract}
Introduction: Diabetes has various impacts on human body. It is thought that diabetes is predisposed by obesity. Obesity may due to several factors including geneticallyenvironmental factors. The recent views that viruses may act as etiology for obesity.

Study objectives: The main objectives of the present study were to investigate the possibility that CMV and HPV of having a role in initiating episodes of obesity and diabetes, and to test the hypothesis that co-existence of multi-viruses including corona virus may work synergistically to increase the impact of COVID-19 on diabetic patients.
\end{abstract}

Methodology: In this study, a diabetic model was induced, the localization of HPV and CMV was determined using immunohistochemistry.

Results: Study findings showed that both viruses HPV and CMV exist in the adipose tissue of diabetic rats. Both viruses were brown in color.

Conclusions: Taken together, both CMV and HPV exist in the adipose tissue of diabetic rats, and this may explain the phenomenon of autoimmunity in diabetes from one side and from another side, we may explain the occurrence of synergistic effects of COVID-19 virus and the other viruses mentioned in this study.

Keywords: diabetes, obesity, viruses, CMV, HPV
Volume 10 Issue 3 - 2020

\author{
Ahed J Alkhatib \\ Department of Legal Medicine, Jordan University of Science \& \\ Technology, Jordan
}

Correspondence: Ahed J Alkhatib, Department of Legal Medicine, Toxicology and Forensic Medicine, Jordan University of Science \& Technology, Jordan, Email ajalkhaib@just.edu.jo

Received: June 08, 2020 | Published: June 23, 2020
Abbreviations: PBS, phosphate buffer saline; BSA, bovine serum albumin

\section{Introduction}

Obesity is considered the fifth driving risk factor for worldwide death, and advances the improvement of a few ceaseless diseases, such as cardiovascular ailments, malignant growth, and diabetes. ${ }^{1-4}$ The worldwide prevalence of obesity has drastically expanded in the course of the most recent 40 years, from $3.2 \%$ in 1975 to $10.8 \%$ in 2014 in men, and from 6.4\% to $14.9 \%$ in women. In the Middle East and North Africa (MENA) area, obesity pervasiveness outperformed $30 \%$ in $2014 .{ }^{4}$ Studies exploring the reasons beyond the spreading of obesity in the MENA locale have for the most part engaged on the function of sociocultural factors including dietary, way of life and physical movement as well as genetic factors. Alongside the increasing ascent in obesity has come a fast ascent in diabetes in the MENA locale, which now has the second most elevated predominance in the world. ${ }^{5}$ In the course of the most recent 20 years, there has been amassing proof supporting the speculation that viral contaminations might be related with obesity in animals and people. Several agents of infectious origin have been associated with obesity in animals and humans: canine distemper infection, Rous related infection, type 7 (RAV-7), Born infection, scrapie specialists, SMAM-1 avian adenovirus, human adenovirus-5, human adenovirus-37, and human adenovirus-36 (Adv36) (Atkinson, 2007). However, adenoviruses are the main irresistible agents to be connected with adiposity in both test animal models and human studies. ${ }^{6}$ Three human adenoviruses have been identified with obesity, with adenovirus 36 being the most considered serotype. ${ }^{7-9}$ Bioinformatics correlations have identified significant differences among Adv36 and other human adenoviruses, recommending one of kind elements of Adv36 that can be connected with fat tissue. ${ }^{10}$ In people, the relationship of common Adv36 infection with the improvement of weight has been involved in a few investigations across different ethnic populaces, in the two grown-ups and kids. Relationship of Adv36 infection with obesity in people was first announced from a US population. ${ }^{11}$

\section{Study objectives}

The main objectives of the present study are to investigate the possibility that CMV and HPV of having a role in initiating episodes of obesity and diabetes, and to test the hypothesis that co-existence of multi-viruses including corona virus may work synergistically to increase the virulence of COVID-19.

\section{Methods}

\section{Preparation of animals and diabetic model}

A total of 20 albino female rats were selected at random bases, and then categorized into control group $(\mathrm{N}=10)$, and diabetic group $(\mathrm{N}=10)$. We conducted this study at the department of biology that is hosting animal house unit in Yarmouk University, Jordan. According to instructions of the university, the study was approved by institutional review board (IRB). Animals were weighted before the starting of the examination. The mean of animal's weight was $185 \pm 7.3$ grams. A 
private room in the animal house was facilitated for this investigation where animals were set in confines. One week before the conduction of research, all animals were treated as the equivalent and were presented to the equivalent ecological conditions for acclimatization purposes. Diabetes was induced in rats utilizing one portion of alloxan monohydrate (Sigma-Aldrich) through intraperitoneal infusion (120 $\mathrm{mg} / \mathrm{kg}$ ) following 12 hour fasting. Blood glucose was estimated day by day to guarantee that animals were hyperglycemic $(\geq 200 \mathrm{mg} / \mathrm{dl})$. All animals were terminated toward the finish of the examination. We have built up our conventions for immunohistochemistry in our research center and recently distributed them. ${ }^{12-14}$

\section{Immunohistochemistry protocols}

Uterus tissue samples were processed using auto-processor that circulated overnight starting from formalin (10\%) for more fixative purposes, then dehydration phase started by passing through $70 \%$ ethanol, $80 \%$ ethanol, $90 \%$ ethanol, $100 \%$ ethanol, then paraffin filtration process started with xylene, xylene and paraffin liquid, and paraffin liquid. Using embedding station (Leica), embedding cassettes were used to obtain paraffin blocks, that were followed by trimming and sectioning using rotatory microtome (Leica). Sections $(3 \mu)$ were cut and mounted on charged slides. Sections were then passed from deparaffinization till water. An oven adjusted at $60 \mathrm{C}$ was used to dissolve paraffin, then slides were put in xylene ( 2 changes, 5 min each), then the hydration phase started with placing sections in $100 \%$ ethanol, $90 \%$ ethanol, $80 \%$ ethanol, $70 \%$ ethanol, and distilled water.

Immunohistochemistry staining protocols started by placing sections in a container containing a solution of $1 \%$ hydrogen peroxide(in absolute methanol)for 15 minutesto block the endogenous activity of peroxidase enzyme. This was followed by washing with phosphate buffer saline (PBS) $(\mathrm{pH}=7.2-7.4)$, and incubation with $1 \%$ bovine serum albumin (BSA) for 20 minutes to block non-specific binding. After washing with PBS, primary monoclonal antibody solution (CMV, 1:100; or HPV, 1:100), Santa Cruz Biotechnology) was incubated with sections for $1 \mathrm{hr}$ using a humid chamber. This was followed by washing with PBS, and then incubated with secondary biotinylated antibodies for 20 minutes, washed with PBS, and then incubated with streptavidin conjugated with horseradish peroxidase enzyme for 20 minutes, washed by PBS. Immunohistochemically reactions were viewed through the reaction with DAB (diaminobenzidine) until the reaction had developed (brown color), and the reaction was finished by washing with tab water. Hematoxylin was used as a counterstain for 30 seconds, then sections were dehydrated through passing into $70 \%$ ethanol, $80 \%$ ethanol, $90 \%$ ethanol, $100 \%$ ethanol, xylene, and mounted with mounting medium.

\section{Results}

\section{Localization of CMV and HPV}

The results of this study showed that both CMV and HPV were existed in the adipose tissue of uterus of diabetic rats. We depended on qualitative methods in which it was enough to detect the existence of the CMV and HPV.Cells containing CMV, or HPV were brown in color against blue background (hematoxylin as counterstain). Cells were identified in adipose tissue included in the uterus.

\section{Discussion}

Although the results of this study are not large, but they are important for several reasons: the results of the present study pointed to the importance of adipose tissue in diabetes which is in line with other studies. ${ }^{1-5}$ The results supported other studies that tried to correlate obesity with viruses. ${ }^{3}$ Previous studies depended on measuring antiviral antibodies against viruses on blood, which although it seems good, but still indirect. In this study, we detected viral cells in adipose tissue itself which gives a direct clue to establish a causal relationship between obesity and diabetes with viral implications. Viruses involved in the obesity include a group of viruses such as SMAM1 avian adenovirus, human adenovirus-5, human adenovirus-37, and human adenovirus-36 (Adv36). ${ }^{3}$ This study added two viruses that were not previously considered: CMV and HPV. This means that the umbrella of viral involvement is being expanded and may shift the understanding of diabetes towards new levels that exceed the classic pattern of diabetes involving genetic susceptibility and other environmental factors. ${ }^{5}$

The idea of having autoimmune diseases to explain diabetes from initiation and progression may be supported by the implication of viruses in diabetes. ${ }^{15}$ The idea of having great impacts of COVID-19 on diabetic patients are varied including infection susceptibility, immunity defects. ${ }^{16-20} \mathrm{I}$ think that there is another dimension that may explain the impact of COVID-19 on diabetic patients through the synergistic interactions between viruses such as HPV and CMV with COVID-19 virus.

\section{Conclusions}

The present study showed that both CMV and HPV exist in the adipose tissue of diabetic rats. Study findings showed that the existence of these viruses may explain the phenomenon of autoimmunity in diabetes from one side and from another side, we may explain the occurrence of synergistic effects of COVID-19 virus and the other viruses mentioned in this study.

\section{Acknowledgments}

None.

\section{Conflicts of interest}

The author declares that there is no conflict of interest.

\section{Funding}

None.

\section{References}

1. Bray GA, Champagne CM. Beyond energy balance: there is more to obesity than kilocalories. J Am Diet Assoc. 2005;105(suppl 1):S17-S23.

2. Bray GA, Bellanger T. Epidemiology, trends, and morbidities of obesity and the metabolic syndrome. Endocrine. 2006;29:109-117.

3. Atkinson RL. Viruses as an Etiology of Obesity. Mayo Clinic Proceedings. 2007;82(10):1192-1198.

4. Nader Lessan, Koramannil R Saradalekshmi, Budour Alkaf, et al. Obesity and Diabetes in an Arab population: Role of Adenovirus 36 Infection. Scientific Reports. 2020;10:8107.

5. Abuyassin, B, Laher I. Diabetes epidemic sweeping the Arab world. World journal of diabetes. 2016;7:165.

6. Ahed Al-khatib. Co-expression of inos and hsp70 in diabetes type 1 makes a rational hypothesis to explain the diabetic neuropathy. European Scientific Journal. 2013;9(3):1857-7881. 
7. Ahed J AlKhatib, Fatima Laiche, Mosleh A Alkhatatbeh, et al. Leaf extract of U. Pilulifera downregulates the expression of INOs in kidneys of diabetic rats. European Scientific Journal. 2014;10(21):302-309.

8. Akheruzzaman M, Hegde V, Dhurandhar NV. Twenty-five years of research about adipogenic adenoviruses: A systematic review. Obesity Reviews. 2019;20:499-509.

9. Arnold J. Genomic characterization of human adenovirus 36, a putative obesity agent. Virus research. 2010;149:152-161.

10. Atkinson RL. Human adenovirus-36 is associated with increased body weight and paradoxical reduction of serum lipids. International Journal of Obesity. 2004;29:281.

11. de Candia P, Prattichizzo F, Garavelli S, et al. Type 2 Diabetes: How Much of an Autoimmune Disease? Frontiers in endocrinology. 2019;10:451.

12. Dhurandhar N. Increased adiposity in animals due to a human virus. International journal of obesity. 2000;24:989.

13. Guan WJ, Ni ZY, Hu Y, et al. Clinical characteristics of coronavirus disease 2019 in China. $N$ Engl J Med. 2020.

14. Liqaa A Raffee, Khaled Zayed Alawneh, Ahed Jumah Al-Khatib, et al Overexpression of HSP90 in Skin of Diabetic Rats Impacts Wound Healing Process. Research Journal of Biological Sciences. 2016;11:63-66.
15. Ruan Q, Yang K, Wang W, et al. Clinical predictors of mortality due to COVID-19 based on an analysis of data of 150 patients from Wuhan, China. Intensive Care Med. 2020; 46(5):846-848.

16. Vangipuram S. Adipogenic human adenovirus-36 reduces leptin expression and secretion and increases glucose uptake by fat cells. International journal of obesity. 2007;31:87.

17. Whigham LD, Israel BA, Atkinson RL. Adipogenic potential of multiple human adenoviruses in vivo and in vitro in animals. American Journal of Physiology-Regulatory, Integrative and Comparative Physiology. 2006;290:R190-R194.

18. Yang J, Zheng Y, Gou X, et al. Prevalence of comorbidities in the novel Wuhan coronavirus (COVID-19) infection: a systematic review and metaanalysis. Int J Infect Dis. 2020;94:91-95.

19. Yang X, Yu Y, Xu J, et al. Clinical course and outcomes of critically ill patients with SARS-CoV-2 pneumonia in Wuhan, China: a singlecentered, retrospective, observational study. Lancet Respir Med. 2020;8(5):475-481.

20. Zhang JJ, Dong X, Cao YY, et al. Clinical characteristics of 140 patients infected with SARS-CoV-2 in Wuhan, China. Allergy. 2020. 